\title{
HIV Testing and Outcomes Among Hispanics/Latinos - United States, Puerto Rico, and U.S. Virgin Islands, 2014
}

Shubha Rao, MPH${ }^{1}$; Puja Seth, $\mathrm{PhD}^{1}$; Tanja Walker, MPH${ }^{1}$; Guoshen Wang, MS¹; Mesfin S. Mulatu PhD ${ }^{1}$; John Gilford, PhD ${ }^{1}$; Emilio J. German, MSHSA ${ }^{2}$

The 2015 National HIV/AIDS Strategy provides an updated plan to address health disparities in communities at high risk for human immunodeficiency virus (HIV) infection $(1,2)$. Hispanics/Latinos* are disproportionately affected by HIV in the United States. In 2014, 23\% of HIV diagnoses were among Hispanics/Latinos, who represented $16 \%$ of the U.S. population (3). To examine HIV testing services, CDC analyzed 2014 data from the National HIV Prevention Program Monitoring and Evaluation (NHM\&E) system submitted by 60 CDCfunded health departments ${ }^{\dagger}$ and 151 community-based organizations. Among Hispanics/Latinos tested, gay, bisexual, and other men who have sex with men (MSM) had the highest percentage of HIV diagnoses (2\%). MSM accounted for 19.8\% of HIV test events conducted among Hispanics/Latinos and 63.8\% of Hispanics/Latinos who received an HIV diagnosis in non-health care settings. ${ }^{\S}$ Approximately $60 \%$ of Hispanics/ Latinos who received an HIV diagnosis were linked to HIV medical care within 90 days; this percentage was lower in the South than in other U.S. Census regions. HIV prevention programs that are focused on expanding routine HIV screening and targeting and improving linkage to medical care and other services (e.g., partner services) for Hispanics/Latinos can help identify undiagnosed HIV cases and reduce HIV transmission.

CDC analyzed NHM\&E HIV test event-level data submitted by $60 \mathrm{CDC}$-funded testing programs at health departments and 151 community-based organizations. Data were stratified by age, gender, U.S. Census region, HIV

\footnotetext{
* Persons who are Hispanic/Latino can be of any race.

$\dagger 50$ states, the District of Columbia, Puerto Rico, the U.S. Virgin Islands, and eight Metropolitan Statistical Areas or specified Metropolitan Divisions: Baltimore, Chicago, Fulton County (Atlanta), Houston, Los Angeles County, New York City, Philadelphia, and San Francisco. In 2014, one health department submitted aggregate-level data, and therefore, was excluded from the analysis.

${ }^{\S}$ Data to identify target populations are required for all test events conducted in non-health care settings and are only required for HIV-positive persons from health care settings. Therefore, data for target populations were only from tests conducted in non-health care settings ( $\mathrm{N}=879,063$ for all CDCfunded test events and $\mathrm{N}=197,802$ for Hispanics/Latinos). Health care settings include inpatient facilities, outpatient facilities, and emergency departments. Non-health care settings include HIV counseling and testing sites and community settings.

An HIV test event is a sequence of one or more HIV tests conducted to determine a person's HIV status. During one testing event, a person might be tested once (e.g., one rapid test or one conventional test) or multiple times (e.g., one rapid test followed by one conventional test to confirm a preliminary HIV-positive test result).
}

prevalence, ${ }^{* *}$ and certain target populations (i.e., MSM, persons who inject drugs, heterosexual males, and heterosexual females)..$^{\dagger \dagger}$ Data to identify target populations are required for all test events conducted in non-health care settings and are only required for HIV-positive persons from health care settings. Target population data included in the analysis were only from non-health care settings. Valid HIV test events were tests for which the results (i.e., positive or negative) were known. Analyses included persons who tested positive for HIV during the most current test event in 2014 and were not previously reported in the health department's HIV surveillance system. Clients' self-reported data regarding previous HIV status were used for grantees who were not able to verify earlier test results within the grantee's surveillance system. The outcomes among Hispanics/Latinos with HIV infection diagnosed in 2014 included linkage to HIV medical care within any timeframe (e.g., attendance at first medical care appointment); linkage to HIV medical care within 90 days; interview for partner services (i.e., soliciting information from HIV-positive persons about their sex partners and drug-injecting partners, who can then be notified of their potential exposures and offered services to protect their health) (4); and referral to HIV risk-reduction services (i.e., services aimed at reducing the risk for HIV transmission, such as behavioral interventions and risk-reduction counseling. HIV risk-reduction services exclude HIV posttest counseling, mental health services, and housing needs) (5).

During 2014, a total of 3,049,845 CDC-funded HIV test events were conducted in the United States, Puerto Rico, and the U.S. Virgin Islands. Hispanics/Latinos accounted for $22.6 \%(687,777)$ of all test events conducted, and for $25.3 \%$ and $25.8 \%$ of HIV test events among persons aged $30-39$ years and $40-49$ years, respectively, as well as $31.4 \%$

\footnotetext{
** HIV prevalence is defined based on the number of persons living with diagnosed HIV infection (PLWH) in 2013. The jurisdictions are classified based on HIV prevalence as follows: high, $\geq 20,000$ PLWH; medium, 4,000-19,999 PLWH; medium-low, 1,000-3,999 PLWH; and low, <1,000 PLWH.

$\dagger \dagger$ MSM include males who reported male-to-male sexual contact as well as males who reported both male-to-male sexual contact and injection drug use in the past 12 months. Persons who inject drugs include persons who reported injection drug use in the past 12 months. Heterosexual males include males who only reported heterosexual contact with a female in the past 12 months. Heterosexual females include females who only reported heterosexual contact with a male in the past 12 months.
} 
of HIV test events among transgender persons. By census region, Hispanics/Latinos accounted for $>90 \%$ of all test events conducted in the U.S. dependent areas of Puerto Rico and the U.S. Virgin Islands (98\% were conducted in Puerto Rico) and $40.1 \%$ of test events in the West census region. Among all HIV test events that occurred in medium-low HIV prevalence and high HIV prevalence areas, Hispanics/Latinos accounted for $22.8 \%$ and $27.0 \%$, respectively. Hispanic/Latino MSM accounted for $24.4 \%$ of all HIV test events conducted among MSM in non-health care settings (Table 1).
Hispanics/Latinos accounted for $23.3 \%$ (2,906) of all persons who received a diagnosis of HIV in 2014. Hispanics/Latinos accounted for $27.4 \%$ and $26.8 \%$ of diagnoses among persons aged $30-39$ years and $40-49$ years, respectively; $24.7 \%$ of diagnoses among males; and $23.6 \%$ of diagnoses among transgender persons. Almost all diagnoses in the U.S. dependent areas and $36.0 \%$ of diagnoses in the West were among Hispanics/Latinos. Among persons in medium-low and high prevalence areas, Hispanics/Latinos accounted for $25.7 \%$ and $24.7 \%$ of diagnoses, respectively. Hispanics/Latinos accounted for $25.3 \%$ of all

TABLE 1. Number and percentage of HIV test events and diagnoses among Hispanics/Latinos, by demographic characteristics and target populations, compared with all CDC-funded HIV test events - United States, Puerto Rico, and U.S. Virgin Islands, 2014

\begin{tabular}{|c|c|c|c|c|c|c|}
\hline \multirow[b]{3}{*}{ Characteristic } & \multicolumn{3}{|c|}{ HIV test events* } & \multicolumn{3}{|c|}{ HIV diagnoses ${ }^{\dagger}$} \\
\hline & \multirow{2}{*}{$\begin{array}{c}\text { All CDC-funded } \\
\begin{array}{c}\text { valid HIV test } \\
\text { events }\end{array} \\
\text { No. }\end{array}$} & \multicolumn{2}{|c|}{ Among Hispanics/ Latinos } & \multirow{2}{*}{$\begin{array}{c}\begin{array}{c}\text { Total } \\
\text { HIV diagnoses }\end{array} \\
\text { No. }\end{array}$} & \multicolumn{2}{|c|}{ Among Hispanics/ Latinos } \\
\hline & & No. & (\%) & & No. & (\%) \\
\hline \multicolumn{7}{|l|}{ Age group (yrs) } \\
\hline $13-19$ & 237,873 & 49,095 & $(20.6)$ & 420 & 86 & $(20.5)$ \\
\hline $20-29$ & $1,213,767$ & 250,200 & $(20.6)$ & 5,505 & 1,175 & $(21.3)$ \\
\hline $30-39$ & 702,686 & 177,843 & $(25.3)$ & 2,999 & 821 & $(27.4)$ \\
\hline $40-49$ & 420,579 & 108,611 & $(25.8)$ & 1,917 & 514 & $(26.8)$ \\
\hline$\geq 50$ & 448,512 & 97,065 & $(21.6)$ & 1,601 & 309 & $(19.3)$ \\
\hline \multicolumn{7}{|l|}{ Gender } \\
\hline Male & $1,541,082$ & 334,932 & $(21.7)$ & 10,208 & 2,521 & $(24.7)$ \\
\hline Female & $1,484,902$ & 348,593 & $(23.5)$ & 2,038 & 342 & $(16.8)$ \\
\hline Transgender & 11,469 & 3,601 & (31.4) & 174 & 41 & $(23.6)$ \\
\hline \multicolumn{7}{|l|}{ U.S census region } \\
\hline Northeast & 508,162 & 129,343 & $(25.5)$ & 2,035 & 555 & $(27.3)$ \\
\hline Midwest & 373,576 & 37,292 & (10.0) & 1,558 & 139 & (8.9) \\
\hline South & $1,654,904$ & 290,223 & (17.5) & 6,893 & 1,303 & $(18.9)$ \\
\hline West & 464,481 & 186,147 & $(40.1)$ & 1,673 & 602 & $(36.0)$ \\
\hline U.S. dependent areas & 48,722 & 44,772 & (91.9) & 313 & 307 & $(98.1)$ \\
\hline \multicolumn{7}{|l|}{ HIV prevalence ${ }^{\S}$} \\
\hline High & $1,843,344$ & 496,795 & $(27.0)$ & 8,362 & 2,067 & $(24.7)$ \\
\hline Medium & $1,108,376$ & 170,803 & (15.4) & 3,792 & 762 & $(20.1)$ \\
\hline Medium-low & 79,027 & 18,031 & $(22.8)$ & 284 & 73 & $(25.7)$ \\
\hline Low & 19,098 & 2,148 & $(11.2)$ & 34 & 4 & $(11.8)$ \\
\hline \multicolumn{7}{|c|}{ Target populations (non-health care settings only) q, $* *^{*}$} \\
\hline Men who have sex with men & 160,499 & 39,122 & $(24.4)$ & 3,083 & 780 & $(25.3)$ \\
\hline Persons who inject drugs & 35,453 & 6,021 & $(17.0)$ & 126 & 28 & $(22.2)$ \\
\hline Heterosexual males & 184,915 & 37,112 & $(20.1)$ & 557 & 116 & $(20.8)$ \\
\hline Heterosexual females & 201,786 & 40,077 & (19.9) & 483 & 66 & (13.7) \\
\hline Total & $3,049,845$ & 687,777 & $(22.6)$ & 12,472 & 2,906 & $(23.3)$ \\
\hline
\end{tabular}

Abbreviation: HIV = human immunodeficiency virus.

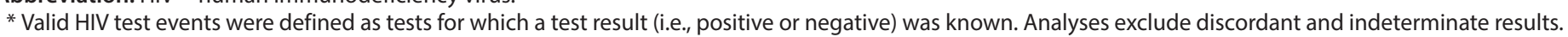

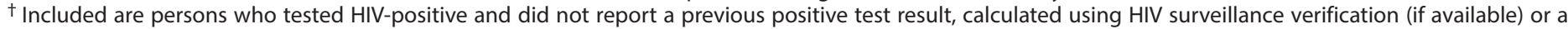
person's self-reported previous HIV status.

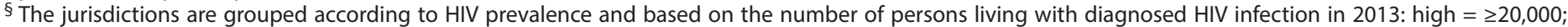
medium = 4,000-19,999; medium-low =1,000-3,999; and low $=<1,000$.

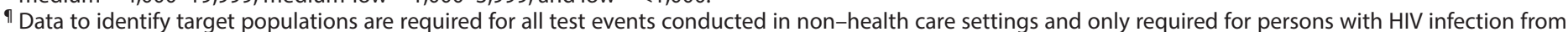

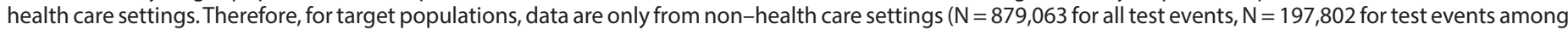

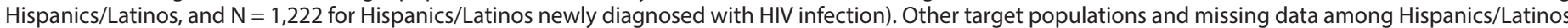

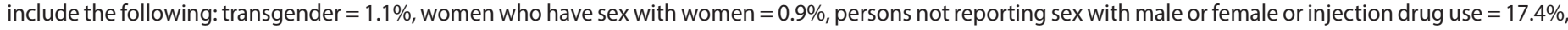
and missing/invalid $=18.8 \%$.

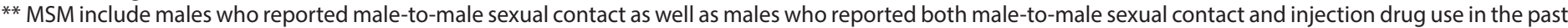

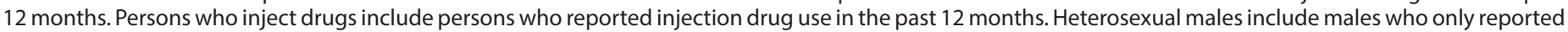

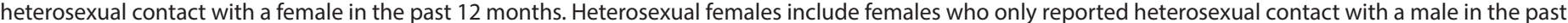
12 months. 
MSM and $22.2 \%$ of all persons who inject drugs receiving an HIV diagnosis in non-health care settings (Table 1).

Overall, 36.4\% of 687,777 test events among Hispanics/ Latinos were among persons aged $20-29$ years; $50.7 \%$ were among females; $42.2 \%$ were among persons residing in the South census region; and $72.2 \%$ were among persons residing in high HIV prevalence areas. In non-health care settings, MSM accounted for $19.8 \%$ of all HIV test events conducted among Hispanics/Latinos (Table 2).

HIV-positivity among Hispanics/Latinos tested in 2014 was $0.4 \%$. Among Hispanics/Latinos tested in non-health care settings, HIV-positivity was highest among MSM (2.0\%), followed by persons who inject drugs $(0.5 \%)$. MSM accounted for $19.8 \%(39,122$ of 197,802$)$ of HIV test events conducted among Hispanics/Latinos in non-health care settings, and 63.8\% (780 of 1,222) of Hispanics/Latinos who received an HIV diagnosis in non-health care settings. Among Hispanics/Latinos who received a diagnosis, $71.0 \%$ were linked to HIV medical care within any timeframe; $61.1 \%$ were linked to medical care within 90 days, $60.0 \%$ were interviewed for HIV partner services, and $64.0 \%$ were referred to risk-reduction services. The percentage of Hispanics/Latinos linked to HIV medical care within 90 days or referred to partner services or risk-reduction services was lower in the South than in other census regions. More than $60.0 \%$ of Hispanic/Latino MSM tested in non-health care settings were linked to HIV medical care or referred to partner services or risk-reduction services (Table 2).

\section{Discussion}

Hispanics/Latinos in the United States are disproportionately affected by HIV. In 2014, the rate of HIV diagnosis among Hispanics/Latinos (18.4 per 100,000 population) was approximately three times that of non-Hispanic whites (6.1 per 100,000 population), but less than that of non-Hispanic blacks/African Americans (49.4 per 100,000 population) (3). The findings in this analysis indicate that Hispanics/Latinos accounted for $22.6 \%$ of all HIV test events and $23.3 \%$ of HIV diagnoses. MSM accounted for $19.8 \%$ of HIV test events conducted among Hispanics/Latinos, and MSM represented 63.8\% of Hispanics/Latinos who received an HIV diagnosis in non-health care settings.

Although $61.1 \%$ of Hispanics/Latinos were linked to HIV medical care within 90 days of diagnosis, that is below the goal established by the 2010 National HIV/AIDS Strategy to link $85.0 \%$ of persons within 90 days of diagnosis (1). Compared with whites, Hispanics/Latinos are less likely to be linked to care $(83.9 \%$ versus $87.1 \%)$ and achieve viral suppression $(54.2 \%$ versus $62.0 \%$ ) (G), which suggests that improved strategies are needed to increase linkage among Hispanics/Latinos as a critical step in achieving viral suppression. Effective public health

\section{Summary}

What is already known about this topic?

In 2014, $23 \%$ of HIV diagnoses were among Hispanics/Latinos, who made up $16 \%$ of the U.S. population. In 2014, the rate of HIV diagnoses among Hispanics/Latinos was approximately three times that for non-Hispanic whites (18.4 compared with. 6.1 per 100,000 population).

What is added by this report?

The analysis showed that Hispanics/Latinos accounted for $22.6 \%$ of CDC-funded HIV testing events and $23.3 \%$ of persons with newly diagnosed HIV infection. The percentages of Hispanics/Latinos linked to HIV medical care, interviewed for partner services, and referred to prevention services were lower in the South compared with other U.S. census regions.

Approximately $60 \%$ of Hispanics/Latinos were linked to medical care within 90 days of diagnosis, which is below the $85 \%$ goal of the National HIV/AIDS Strategy.

What are the implications for public health practice?

The findings emphasize the need for culturally and linguistically sensitive prevention strategies to promote diagnosis of HIV infection and linkage to medical care among Hispanics/Latinos to improve health outcomes. Stakeholders and partners should focus on prevention and care strategies for Hispanic/Latino subpopulations (e.g., men who have sex with men and persons who inject drugs) to reduce HIV-related disparities.

strategies also are needed to strengthen partner services and increase referrals for risk-reduction services among Hispanics/ Latinos, especially among Hispanics/Latinos in the South. Given these data, prevention efforts that include targeted specific geographic regions might increase early diagnosis and improve HIV-related health outcomes among Hispanics/Latinos (7).

The findings in this report are subject to at least three limitations. First, the findings describe HIV tests conducted at CDC-funded health departments and community-based organizations only and are not representative of all HIV testing in the United States. Second, data on referral and linkage to HIV medical care, partner services, and risk-reduction services represent the minimum percentage (i.e., include records without valid data on the outcome in the denominator) achieved and likely underestimate the percentage referred and linked. Finally, collection of data on target populations is required in all non-health care settings but only for HIV-positive test events in all health care settings; therefore, underreporting the number of test events conducted among target populations.

Broader implementation of routine HIV screening and targeting testing among Hispanics/Latinos, most notably MSM and transgender persons, as recommended by CDC guidelines (8) and the U.S. Preventive Services Task Force (9) is critical to identify persons who are unaware of their HIV status. Programmatic strategies (e.g., HIV preexposure prophylaxis) 
TABLE 2. Linkage to HIV medical care, referral and interview for HIV partner services, and referral to HIV risk-reduction services among Hispanics/ Latinos, by demographic characteristics and target populations - United States, Puerto Rico, and U.S. Virgin Islands, 2014

\begin{tabular}{|c|c|c|c|c|c|c|c|}
\hline & $\begin{array}{l}\text { Valid HIV test } \\
\text { events among } \\
\text { Hispanics/ } \\
\text { Latinos* }\end{array}$ & $\begin{array}{l}\text { Diagnoses } \\
\text { among } \\
\text { Hispanics/ } \\
\text { Latinos }^{\dagger}\end{array}$ & $\begin{array}{c}\text { Linked to HIV } \\
\text { medical care } \\
\text { within any } \\
\text { timeframe }\end{array}$ & $\begin{array}{l}\text { Linked to HIV } \\
\text { medical care } \\
\text { within } 90 \text { days of } \\
\text { diagnosis }\end{array}$ & $\begin{array}{l}\text { Referred to HIV } \\
\text { partner services }\end{array}$ & $\begin{array}{l}\text { Interviewed for } \\
\text { HIV partner } \\
\text { services }\end{array}$ & $\begin{array}{c}\text { Referred to HIV } \\
\text { risk-reduction } \\
\text { services }\end{array}$ \\
\hline Characteristic & No. & No. (\%) & No. $(\%)$ & No. (\%) & No. (\%) & No. (\%) & No. (\%) \\
\hline \multicolumn{8}{|l|}{ Age group (yrs) } \\
\hline $13-19$ & 49,095 & $86(0.2)$ & $61(70.9)$ & $49(57.0)$ & $61(70.9)$ & $46(53.5)$ & $54(62.8)$ \\
\hline $20-29$ & 250,200 & $1,175(0.5)$ & $852(72.5)$ & $736(62.6)$ & $916(78.0)$ & $719(61.2)$ & $776(66.0)$ \\
\hline $30-39$ & 177,843 & $821(0.5)$ & $573(69.8)$ & $494(60.2)$ & $624(76.0)$ & $486(59.2)$ & $518(63.1)$ \\
\hline $40-49$ & 108,611 & $514(0.5)$ & $366(71.2)$ & $310(60.3)$ & $390(75.9)$ & $309(60.1)$ & $329(64.0)$ \\
\hline$\geq 50$ & 97,065 & $309(0.3)$ & $210(68.0)$ & $187(60.5)$ & $234(75.7)$ & $183(59.2)$ & $183(59.2)$ \\
\hline \multicolumn{8}{|l|}{ Gender } \\
\hline Male & 334,932 & $2,521(0.8)$ & $1,813(71.9)$ & 1,552 (61.6) & $1,948(77.3)$ & $1,524(60.5)$ & $1,625(64.5)$ \\
\hline Female & 348,593 & $342(0.1)$ & $216(63.2)$ & $193(56.4)$ & $241(70.5)$ & $196(57.3)$ & $205(59.9)$ \\
\hline Transgender & 3,601 & $41(1.1)$ & $32(78.0)$ & 29 (70.7) & 34 (82.9) & $21(51.2)$ & $28(68.3)$ \\
\hline \multicolumn{8}{|c|}{ U.S. census region } \\
\hline Northeast & 129,343 & $555(0.4)$ & $453(81.6)$ & $433(78.0)$ & $486(87.6)$ & $371(66.8)$ & $489(88.1)$ \\
\hline Midwest & 37,292 & $139(0.4)$ & $85(61.2)$ & $78(56.1)$ & $112(80.6)$ & $85(61.2)$ & $102(73.4)$ \\
\hline South & 290,223 & $1,303(0.4)$ & $900(69.1)$ & $683(52.4)$ & $849(65.2)$ & 725 (55.6) & 569 (43.7) \\
\hline West & 186,147 & $602(0.3)$ & $377(62.6)$ & $335(55.6)$ & $498(82.7)$ & $339(56.3)$ & $432(71.8)$ \\
\hline $\begin{array}{l}\text { U.S. dependent } \\
\text { areas }\end{array}$ & 44,772 & $307(0.7)$ & $248(80.8)$ & $247(80.5)$ & $280(91.2)$ & $223(72.6)$ & $268(87.3)$ \\
\hline \multicolumn{8}{|l|}{ HIV prevalence ${ }^{\S}$} \\
\hline High & 496,795 & $2,067(0.4)$ & $1,438(69.6)$ & $1,186(57.4)$ & $1,492(72.2)$ & $1,145(55.4)$ & $1,189(57.5)$ \\
\hline Medium & 170,803 & $762(0.4)$ & $555(72.8)$ & $523(68.6)$ & $662(86.9)$ & $529(69.4)$ & $605(79.4)$ \\
\hline Medium-low & 18,031 & $73(0.4)$ & 68 (93.2) & $65(89.0)$ & $69(94.5)$ & $67(91.8)$ & $64(87.7)$ \\
\hline Low & 2,148 & $4(0.2)$ & $2(50.0)$ & $2(50.0)$ & $2(50.0)$ & $2(50.0)$ & $2(50.0)$ \\
\hline \multicolumn{8}{|c|}{ Target populations (non-health care settings only) $)^{\eta_{1} * *}$} \\
\hline MSM & 39,122 & $780(2.0)$ & $545(69.9)$ & $519(66.5)$ & $675(86.5)$ & $512(65.6)$ & $520(66.7)$ \\
\hline $\begin{array}{l}\text { Persons who } \\
\text { inject drugs }\end{array}$ & 6,021 & $28(0.5)$ & $15(53.6)$ & $11(39.3)$ & $22(78.6)$ & $12(42.9)$ & $21(75.0)$ \\
\hline $\begin{array}{l}\text { Heterosexual } \\
\text { males }\end{array}$ & 37,112 & $116(0.3)$ & $75(64.7)$ & $72(62.1)$ & $91(78.4)$ & $70(60.3)$ & $70(60.3)$ \\
\hline $\begin{array}{l}\text { Heterosexual } \\
\text { females }\end{array}$ & 40,077 & $66(0.2)$ & 44 (66.7) & 44 (66.7) & $58(87.9)$ & $47(71.2)$ & 39 (59.1) \\
\hline Total & 687,777 & $2,906(0.4)$ & $2,063(71.0)$ & $1,776(61.1)$ & 2,225 (76.6) & $1,743(60.0)$ & $1,860(64.0)$ \\
\hline
\end{tabular}

Abbreviations: HIV = human immunodeficiency virus, MSM = men who have sex with men.

* Valid HIV test events were defined as tests for which a test result (i.e., positive or negative) was known. Analyses exclude discordant and indeterminate results.

+ Included are persons who tested HIV-positive and did not report a previous positive test result, calculated using HIV surveillance verification (if available) or a person's self-reported previous HIV status. The denominator for diagnosed persons is the number of HIV test events. The denominator for linkage, HIV partner services, and HIV risk reduction services is the number of diagnosed persons.

$\S$ The jurisdictions are grouped according to HIV prevalence and based on the number of persons living with diagnosed HIV infection in 2013: high $=\geq 20,000$; medium = 4,000-19,999; medium-low =1,000-3,999; and low $=<1,000$.

" Data to identify target populations are required for all test events conducted in non-health care settings and only required for persons with HIV infection from health care settings. Therefore, for target populations, data are only from non-health care settings ( $\mathrm{N}=879,063$ for all test events, $\mathrm{N}=197,802$ for test events among Hispanics/Latinos, and $\mathrm{N}=1,222$ for Hispanics/Latinos newly diagnosed with HIV infection). Other target populations and missing data among Hispanics/Latinos include the following: transgender $=1.1 \%$, women who have sex with women $=0.9 \%$, persons not reporting sex with male or female or injection drug use $=17.4 \%$, and $\mathrm{missing} / \mathrm{invalid}=18.8 \%$.

** MSM include males who reported male-to-male sexual contact as well as males who reported both male-to-male sexual contact and injection drug use in the past 12 months. Persons who inject drugs include persons who reported injection drug use in the past 12 months. Heterosexual males include males who only reported heterosexual contact with a female in the past 12 months. Heterosexual females include females who only reported heterosexual contact with a male in the past 12 months.

for HIV-negative persons at high risk (especially racial/ethnic minorities and MSM) that complement existing prevention efforts are important to reduce the risk for HIV infection (10). CDC offers bilingual resources to raise awareness about HIV and testing among Hispanics/Latinos, including its newest campaign, Doing It, which is intended to motivate adults to get tested. ${ }^{\$}$ Barriers to accessing and receiving HIV testing among Hispanics/Latinos include lack of health insurance, lower educational status, stigma or fear of discrimination, and fear of disclosing immigration status. 99 HIV prevention

\footnotetext{
$\$ \$$ http://www.cdc.gov/actagainstaids/campaigns/doingit/.

$99 \mathrm{http} / /$ www.cdc.gov/hiv/group/racialethnic/hispaniclatinos/index.html.
} 
programs that focus on culturally and linguistically sensitive prevention strategies to increase testing, enhance linkage to HIV care and partner services, and promote community-level risk-reduction services for Hispanics/Latinos might reduce health disparities and HIV transmission.

\section{Acknowledgments}

Sam Dooley, MD, Janet Heitgerd, PhD, Program Evaluation Branch, Division of HIV/AIDS Prevention, National Center for HIV/ AIDS, Viral Hepatitis, STD, and TB Prevention, CDC; Prevention Program Branch, Quantitative Sciences and Data Management Branch, Division of HIV AIDS Prevention, National Center for HIV/AIDS, Viral Hepatitis, STD, and TB Prevention, CDC.

\footnotetext{
${ }^{1}$ Program Evaluation Branch, Division of HIV/AIDS Prevention, National Center for HIV/AIDS, Viral Hepatitis, STD, and TB Prevention; ${ }^{2}$ Office of Health Equity, Division of HIV/AIDS Prevention, National Center for HIV/ AIDS, Viral Hepatitis, STD, and TB Prevention.

Corresponding author: Shubha Rao, srao1@cdc.gov, 404-639-8521.

\section{References}

1. Office of National AIDS Policy. National HIV/AIDS strategy for the United States. Washington, DC: White House, Office of National AIDS Policy; 2010. https://www.whitehouse.gov/administration/eop/onap/nhas 2. Office of National AIDS Policy. National HIV/AIDS strategy for the United States: updated to 2020. Washington, DC: White House, Office of National AIDS Policy; 2015. https://www.aids.gov/federal-resources/ national-hiv-aids-strategy/nhas-update.pdf
}

3. CDC. Diagnoses of HIV infection in the United States and dependent areas, 2014. HIV surveillance report. Atlanta, GA: US Department of Health and Human Services, CDC; 2015. http://www.cdc.gov/hiv/ library/reports/surveillance

4. CDC. The recommendations for partner services programs for HIV infection, syphilis, gonorrhea, and chlamydial infection. MMWR Recomm Rep 2008 (No. RR-9).

5. CDC, Health Resources and Services Administration, National Institutes of Health, et al. Recommendations for HIV prevention with adults and adolescents with HIV in the United States, 2014. Atlanta, GA: US Department of Health and Human Services, CDC; 2014. https://stacks. cdc.gov/view/cdc/26062

6. CDC. Monitoring selected national HIV prevention and care objectives by using HIV surveillance data-United States and 6 dependent areas, 2014. HIV surveillance supplemental report 2016;21(4). Atlanta, GA: US Department of Health and Human Services, CDC; 2016. http:// www.cdc.gov/hiv/pdf/library/reports/surveillance/cdc-hiv-surveillancesupplemental-report-vol-21-4.pdf

7. CDC. Geographic differences in HIV infection among Hispanics or Latinos-46 states and Puerto Rico, 2010. MMWR Morb Mortal Wkly Rep 2012;61:805-10.

8. Branson BM, Handsfield HH, Lampe MA, et al. Revised recommendations for HIV testing of adults, adolescents, and pregnant women in health-care settings. MMWR Recomm Rep 2006;55(No. RR-14).

9. U.S. Preventive Services Task Force. Human immunodeficiency virus (HIV) infection: screening. Recommendation summary. September 2014.https://www. uspreventiveservicestaskforce.org/Page/Topic/recommendation-summary/ human-immunodeficiency-virus-hiv-infection-screening

10. Smith DK, Van Handel M, Wolitski RJ, et al. Vital signs: estimated percentages and numbers of adults with indications for preexposure prophylaxis to prevent HIV acquisition-United States, 2015. MMWR Morb Mortal Wkly Rep 2015;64:1291-5. http://dx.doi.org/10.15585/ mmwr.mm6446a4 